\title{
Influence of Rotation Velocity Gradient on Line Profiles of Accretion Discs of CVs
}

\author{
D. Korčáková1, T. Nagel ${ }^{2}$, K. Werner ${ }^{2}$, V. Suleimanov ${ }^{2,3}$, \\ and V. Votruba ${ }^{4}$ \\ ${ }^{1}$ Astronomical Institute, Charles University, V Holešovičkách 2, 18000 Praha 8, Czech \\ Republic; email: kor@sirrah.troja.mff.cuni.cz \\ ${ }^{2}$ Institut für Astronomie und Astrophysik, Universität Tübingen, Sand 1, 72076 Tübingen, \\ Germany \\ ${ }^{3}$ Department of Astronomy, Kazan Federal University, Kremlevskaya 18, 420008 Kazan, Russia \\ ${ }^{4}$ Astronomical Institute AV ČR, Fričova 298, 25165 Ondřejov, Czech Republic
}

\begin{abstract}
We show the influence of the Keplerian velocity shear on the line profiles of cataclysmic variable discs. The complete disc structure is taken into account. The radial disc structure follows the alpha disc approximation. Based on this assumption, the vertical structure is computed using the detailed non-LTE code $A c D c$. The obtained opacities and source functions are interpolated in the $2 \mathrm{D}$ grid, where the radiative transfer is calculated with the inclusion of the velocity field gradient.
\end{abstract}

Keywords. cataclysmic variables, accretion disks, line: formation, methods: numerical

\section{Introduction}

The influence of the velocity gradient on the line formation in discs of cataclysmic variables $(\mathrm{CV})$ is usually neglected. However, there are works which show, that this effect can be important for high inclination angles. Nevertheless, some fundamental simplifications had to be used in the past considering the computation efficiency. The current technique allows us to describe the problem in more detail. We try to include the $2 \mathrm{D}$ nature and non-LTE effects in order to evaluate the influence of the rotation velocity gradient on the line formation. A detailed discussion of our results can be found in Korčáková et al. (2011).

\section{Model}

Consistent models of accretion discs are still beyond current computation capabilities. Therefore, we use the following approach. The radial disc structure is calculated using the $\alpha$-disc approximation (Shakura \& Sunyaev 1973). The vertical disc structure and the opacity and source function of the material are obtained from the non-LTE $A c D c$ code (Nagel et al. 2004). The disc is divided into a set of concentric rings, where the radiative transfer equation (RTE), hydrostatic equilibrium and energy balance equations, and nonLTE rate equations are consistently solved using the Accelerated Lambda Iteration (ALI, Werner \& Husfeld 1985). The resulting opacities and source functions are interpolated onto a 2D grid, where the RTE is solved with the inclusion of the velocity field (Korčáková \& Kubát 2005). 

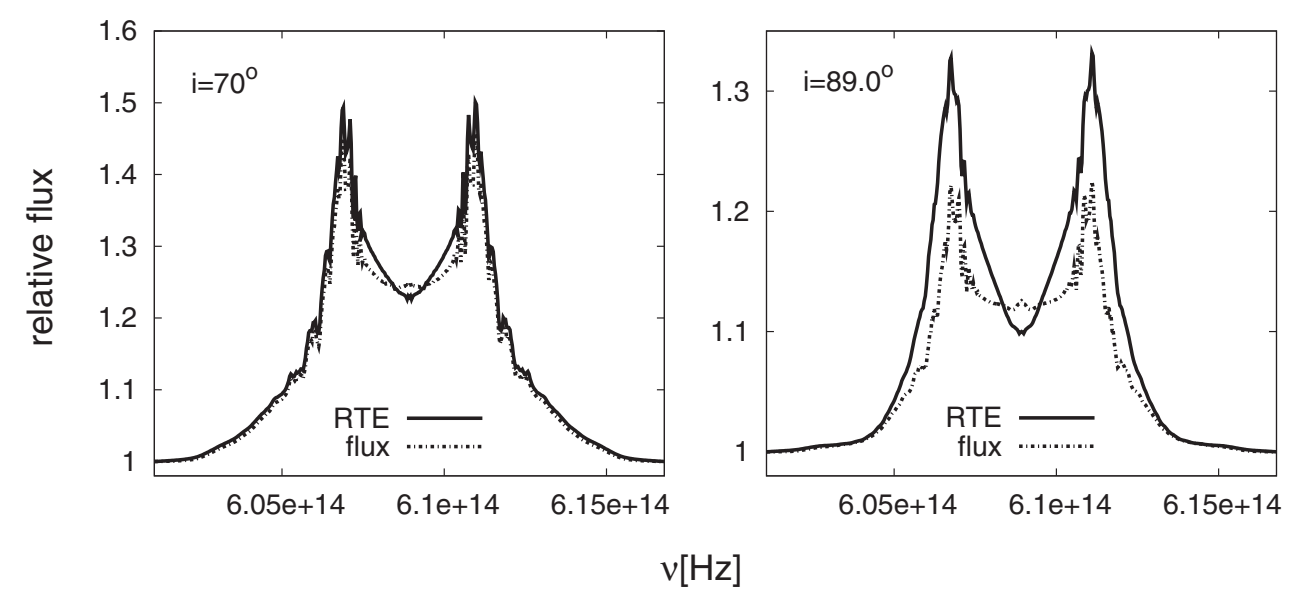

Figure 1. HeI $4923 \AA$ line profiles from accretion discs of an AM CVn system (solid line - the velocity gradient in the RTE, dashed line - the velocity field only in the flux calculation).

\section{Results and conclusion}

The influence of the velocity field on the line profile depends on the ratio of the Doppler shift, caused by macroscopic motion, and line width in the line forming region. Therefore, we investigated the optically thinner quiescent phase of CV discs.

AM CVn systems offer a good possibility to test the velocity shear influence, since the discs are relatively cold, transparent in He lines, and rotation velocity reaches high values $\left(3360 \mathrm{~km} \mathrm{~s}^{-1}\right)$. The results of our calculations are plotted in Fig. 1. The solid line is the solution, where the RTE is solved with the inclusion of the velocity field. The dashed line indicates the result, where the RTE is solved through the static disc and the velocity field is taken into account only in the flux calculation. The line profiles are shown for different inclination angles $i$. Previous works suggested that the change of the line profiles can be important for $i>60^{\circ}$. Our calculations show that even for such an extreme case, the difference is negligible for $i=70^{\circ}$ (Fig. 1, left panel). The change in the line profile can be seen just above the disc edge (Fig. 1, right panel). However, the line-profile differences were negligible for SS Cyg, whose properties are closer to a typical CV disc.

The Keplerian velocity shear can be neglected in many cases. The common approximation, where the radiative transfer is calculated through a static disc and only the outgoing flux incorporates the velocity field, is valid to high accuracy. However, the detailed calculation of the radiative transfer is necessary for optically thin lines in discs seen under high inclination angles and for situations where we see the disc rim.

\section{Acknowledgements}

This research is partly financed by grants $\mathrm{C} 7$ of SFB/Transregio 7 "Gravitational Wave Astronomy," NSh-4224.2008.2, 228398 (the European Commission), 205/09/P476 (GA ČR), and projects MSM0021620860 and AV0Z10030501.

\section{References}

Korčáková, D. \& Kubát, J. 2005, A\&A, 440, 715

Korčáková, D., Nagel, T., Werner, K., Suleimanov, V., \& Votruba, V. 2011, A\&A, 529, 119

Nagel, T., Dreizler, S., Rauch, T., \& Werner, K. 2004, A\&A, 428, 109

Shakura, N. I. \& Sunyaev, R., A. 1973, A\&3A, 24, 337

Werner, K. \& Husfeld, D. 1985, A\&A, 148, 417 